\title{
A colour recognition device for the visually disabled people
}

\author{
Norazila Othman, Mohd Zarhamdy Md. Zain, Iskandar Shah Ishak, Abdul Rahim Abu Bakar, \\ Mastura Ab Wahid, Maziah Mohamad \\ School of Mechanical Engineering, Faculty of Engineering, Universiti Teknologi Malaysia, Malaysia
}

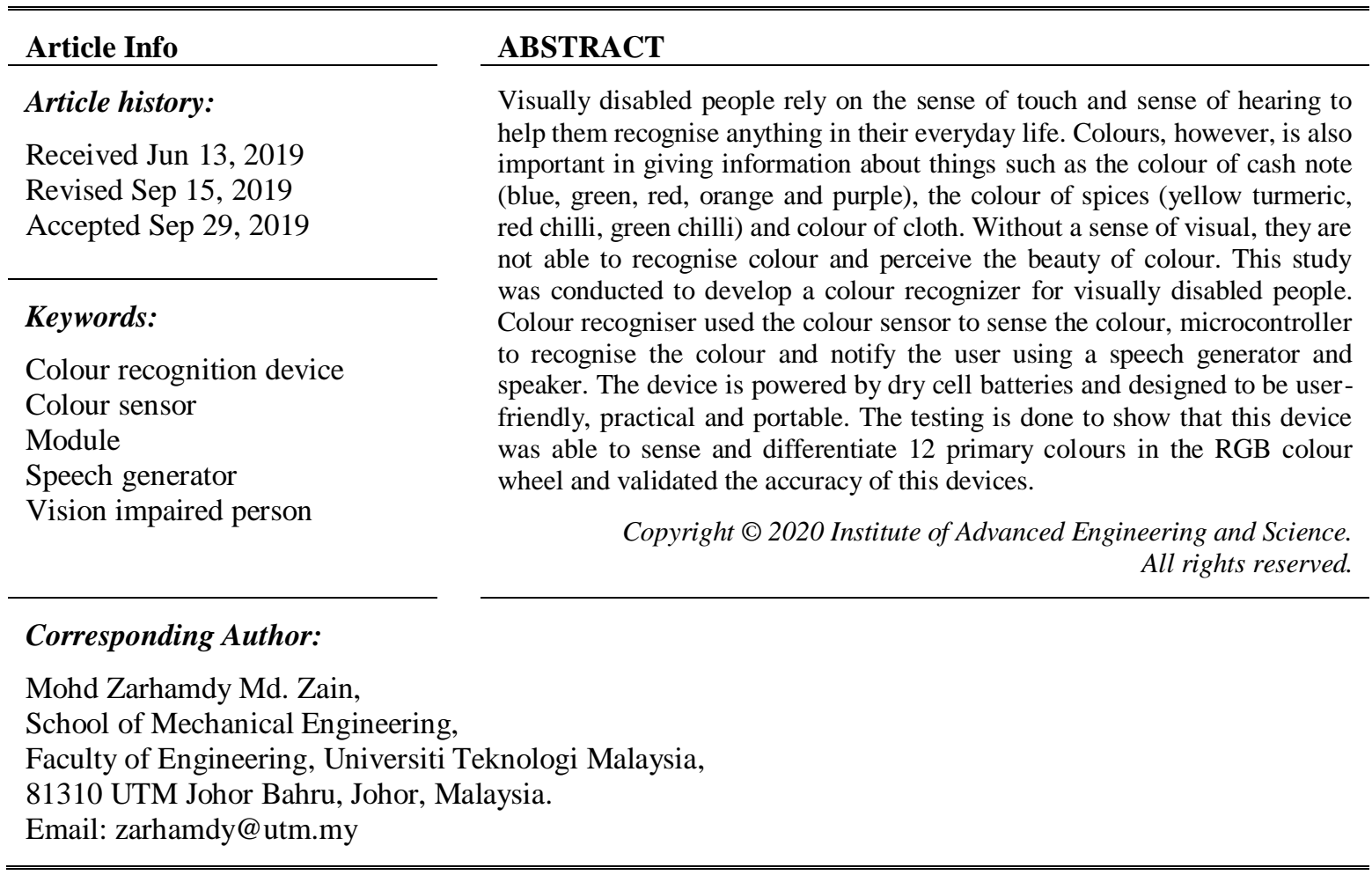

\section{INTRODUCTION}

Nowadays, people with visual disability are about 303 million globally, whereby 39 million of them are considered to be com-pletely blind and the remaining 264 million are having vision limitations or known as partial blindness. About $90 \%$ of them are living in developing countries [1]. As blindness can be defined as a lack of vision, it also can be referred to a loss of vision that cannot be corrected with glasses [2].

Colour is meaningless to people who are born blind as they never see colour and never understand colour. However for some people who become blind later in life or have partial blindness, colour is also important in helping them recognise things. Mostly, visually disabled people face difficulties in recognizing colours in their lives. For instance, to identify colour in pedestrian crossing lights, clothes, paints, foods, and etc. In order to assist the visually disabled person, there have several methods been developed such as Braille with colour code [3] and devices that can produce audio output from a colour sensor data [4-7]. Braille is a form of communication in which people use their fingers to feel elevated bumps on a surface and can understand what is meant to be interpreted [9-14]. Sometime the visually disable people leaves marking such as staples or pin on their cloth to identify colour. By having a colour recognition device they can remove these markings. The developement of a colour recognizer can also help a teacher to teach colours to the visually disable person.

The aim of this study is to develop a device that can detect colours and then inform the colour to the users through the audio output from speakers. Therefore, this device should be simple, user-friendly, practical and affordable to those with vis-ual disability. The objective of this study is achieved by, firstly studying the behaviour of disabled people especially for colour recognition, so it helped in choosing the suitable mechatronics design for colour detection, then prototyping by fabricating the mechatronic design for colour detection and after all of that a verification and evaluation process is carried on 12 primary colours. 
Extensive research on colour recognizer has been performed in the last few years. In India, L. Dhamini1 and D. Bhanuprakash [15] proposed a camera-based sensor and raspberry pi to detect and process the colour. The colour was transformed from the RGB system to the HSL system to better differentiate the colour tone. M. Anil Kumar et al [16] proposed a device connected to a mobile phone that is able to notify the user on the colours. The colour recogniser device and mobile phone are required for this application. The sensor for detecting colours is TCS3200. In Indonesia, Alfa Sheffildi and Rini Fitri [17] developed colour recognition with augmented reality concept based on finger interaction between a user and coloured object. A camera is used for the colour sensor. In Malaysia, Tarek M.D. et al [18] proposed to use a neural network and decision three based machine learning to recognize the colour and give an output to the user through audio and vibration. Three devices were used for the colour recognition system, first, the hand index box that contains the camera, ultrasonic sensor and push button, second, the vibration actuator module and the third is hand wrist box with the processors (raspberry pi). In this paper, a practical single colour recognizer which is a portable, small, lightweight and low cost is proposed to help the visually impaired. The output chosen is an audio output where the user can use an earphone to listen to the colour name. The processor is Arduino Uno and the colour sensor is TCS3200. Before moving on to the methodology, an understanding of the colour system is required and discuss below.

Additive colour system, subtractive colour system and CMYK colour system are the three colour systems available. For the RGB model as shown in Figure 1(a), R for red, G for green and B for blue are the light primaries. Combining these primaries will produce new colours: - green and red light rays produce yellow, blue and green produce cyan, red and blue produce magenta. The RGB (red, green, blue) primary colours when combined in various proportions different colours will be obtained in the visible spectrum. If all primary colours are mixed together white light will be created as in Figure 1(a). In this system, the levels of the three colours red, green and blue can range from 0 to 100 percent of full intensity. Decimal numbers are used to represent each level ranging from 0 to 255, range from 00000000 to 11111111 in binary numbers and from 00 to FF in hexadecimal. the total number of colours is $256 \times 256 \times 256$ which results $16,777,26$ colours. This colour model is normally used for devices employing light such as computer monitors and television sets. Meanwhile, the subtractive colour system is the opposing of additives colour system. It employs cyan, magenta and yellow as the basic primary colour. Combining two of three colours will produce the additives colours and when all three subtractive colours are combined will produce black light. Lastly, the CMYK colour system which means cyan, magenta and, yellow, then become the black colour systems. These four colours are used as the primary colour. The CMYK colour system is used in the printing industry. When these primaries colours are combined, the result is grey [10] as seen in Figure 1(b).

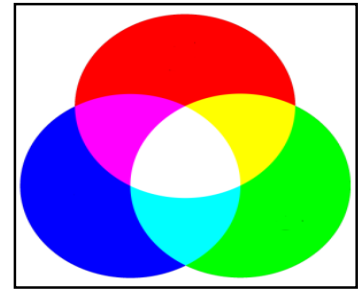

(a)

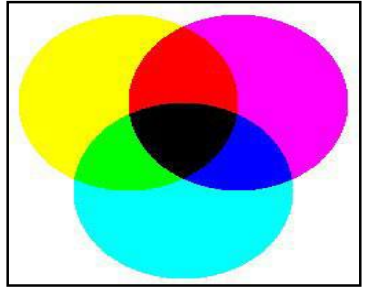

(b)

Figure 1. Colour system. (a) RGB Model. (b) CMYK Model

\section{RESEARCH METHODOLOGY}

\subsection{Hardware}

The electronic components used in the device are Arduino Uno, colour sensor TCS3200, Catalex serial MP3 player and power source. The device casings are modelled using SOLIDWORK software as illustrated in Figure 3 and then they are produced by using a 3D printer machine.

The microcontroller board used is the ATmega328P and called Arduino/Genuino Uno. It has 14 digital input/output pins with 6 PWM pin, 6 Analog inputs, a $16 \mathrm{MHz}$ quartz crystal, a USB connection, a power jack, an ICSP header and a reset button. The microcontroller can be directly connected to a PC using USB cable or by using dry battery to start it.

The colour sensor used is TCS3200. It is a programmable colour light to frequency converter that combines configurable silicon photodiodes and a current-to-frequency converter on a single monolithic CMOS integrated circuit. The TCS3200 reads an $8 * 8$ array of photodiodes, while the output is in a square wave with frequency directly proportional to light intensity (irradiance) as in Figure 2 [12]. 


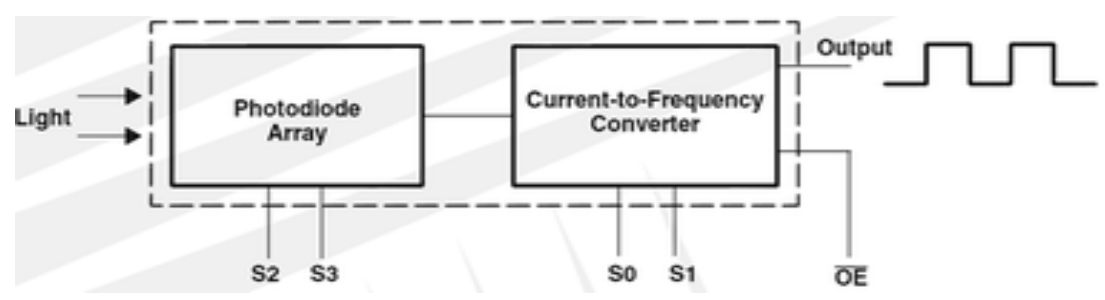

Figure 2. Functional block diagram of TCS 3200

To play the name of the colour, a Catalex MP3 player module is used. It is based on a high-quality MP3 audio chip (YX5300), and support $8 \mathrm{kHz} \sim 48 \mathrm{kHz}$ sampling frequency MP3 and WAV file formats. The audio files for colours name are downloaded into a micro SD and inserted into the TF slot that comes with the Catalex MP3. MCU can control the MP3 playback state by sending commands to the module via UART port, such as switch songs, change the volume and play mode and so on. For a bonus-added feature, the system is designed to make possible for debugging the module via USB to UART module. It is compatible with Arduino / AVR / ARM / PIC.

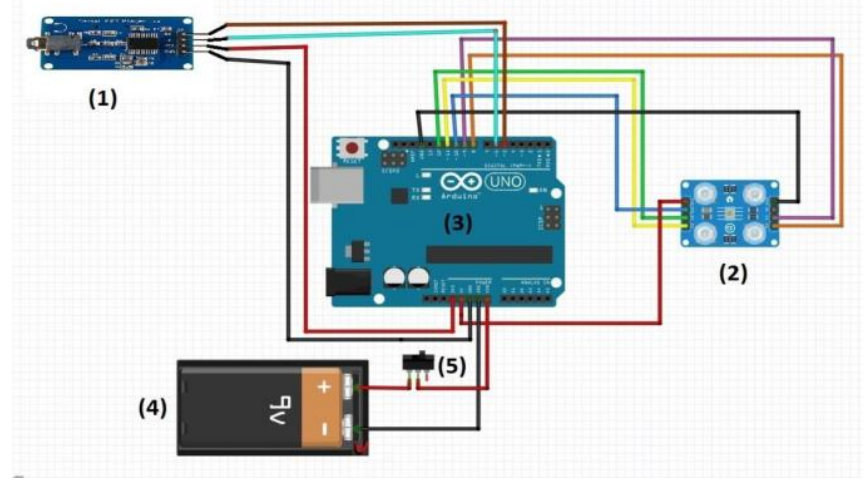

Figure 3. Functional block diagram of TCS 3200

This combination of components is powered up using one unit of a $9 \mathrm{~V}$ dry cell which is placed at the bottom of the device. The circuit diagram of the device is shown in Figure 3 and the components used are: 1. Catalex serial MP3 player, 2. TCS3200 colour sensor, 3. Arduino UNO, 4. 9V dry cell and 5. Toggle switch.

\subsection{Software}

In this section the software used will be discuss and the connection between the TCS3200, Arduino microcontroller and the software. All Software implementation of this device is the Arduino software. The programming code is developed in Arduino software which is then uploaded to Arduino board via USB connector. The first setup is to initilized the integer variables that control the input and output pins of the sensor. Secondly, communication speed is setup to $9600 \mathrm{bps}$ since the MP3 module is configured to that speed and thirdly, the pins which are used to select the filters are define. Once the connection has been made, check whether the information is coming through a serial port or not. Now checks the serial port data that identify which colour the application asks. Then different filter pins are configured based on information given in Table 1.

Table 1. Filter Pin for the Colour Sensor

\begin{tabular}{ccc}
\hline Pin S2 & Pin S3 & Photodiode Type \\
\hline L & L & Red \\
L & H & Blue \\
H & L & Clear \\
H & H & Green \\
\hline
\end{tabular}




\subsection{Prototype of the Device}

At a grace of the 3D printing machine, the prototype is materialized. Then all the electronic components are embedded in it. The completed prototype is then tested and verified to ensure its capability to recognize the colours correctly. Figure 4 shows the prototype of the device.

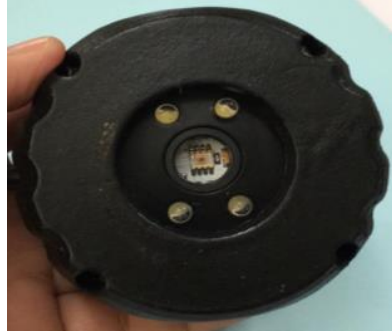

(a)

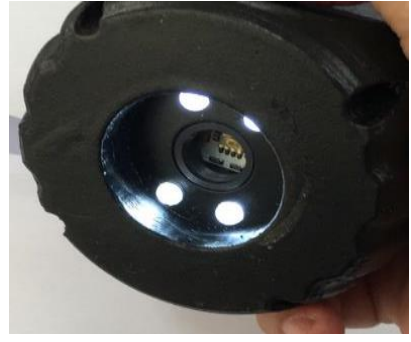

(b)

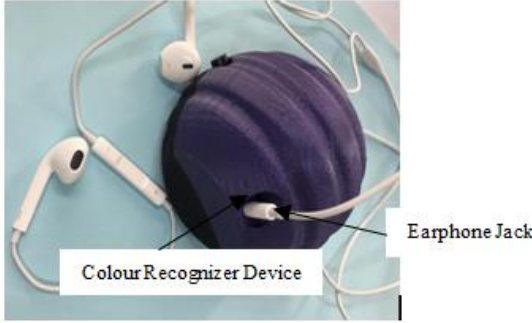

(c)

Figure 4. Prototype of the device. (a) Bottom of the device. (b) Light is turned on the device. (c) The device with speaker/earphone connection

\subsection{Experiment and Verification Test}

The experiment is performed under a room condition with a moderate light source from the environment up to 550 lux. From this test, the reading of the colour of the object will be done by TCS3200 colour sensor with four photodiodes. The detected colours will be converted to a frequency which will then be read by Arduino. The data are acquired and processed to determine its performance. The test is carried out using 12 standard colours which are red, orange, yellow, green yellow, green, green cyan, cyan, blue cyan, blue, blue magenta, magenta and red magenta as in Figure 5. For the experiment, different colour material such as a different colour fabric is used as test items. The colour recognizer must be placed flat on the surface of the fabric or item such as shown in Figure 6.

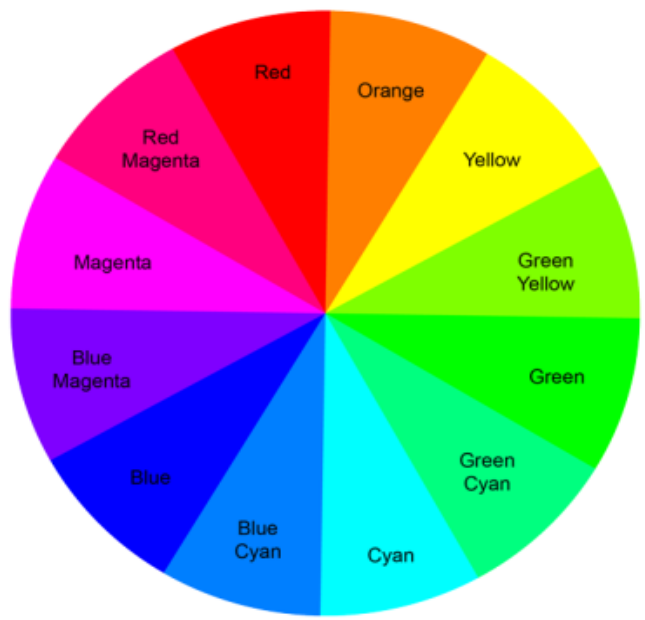

Figure 5. Colour wheel for verification process

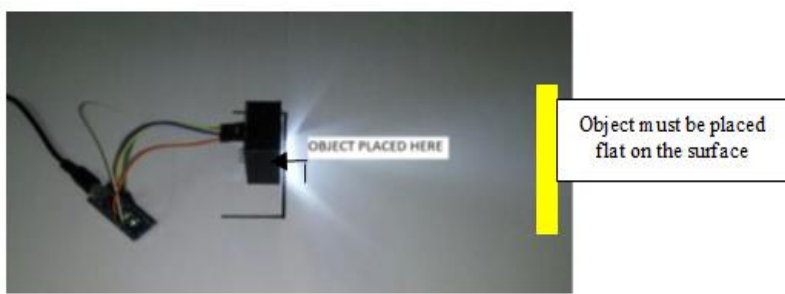

Figure 6. Placement of object for colour recognition

The verification is performed with the aids of the serial monitor of Arduino environment software. The colour reflected by the white light will be transferred to the Arduino board and compared based on the frequency detected by the colour sensor. Initial setup will use the three primaries colours red, blue and green. The maximum and minimum frequency output for each colour will be recorded and converted to RGB values (from 0 to 255). In the programming, the frequency-scaling of colour has been tabulated and compared with frequency detected by the four photodiodes which will then be picked to be vocalized by the colour recognizer sensor. 
Figure 7 shows the initial result for the frequency to RGB mapping. Here the expected frequency when red is the dominant colour is $8 \mathrm{kHz}$ and when blue is the dominant colour, the frequency will be around $11 \mathrm{kHz}$. Verification on real object for the colour recognizer is done on t-shirts. From the testing, it has shown that the colour recognizer is able to detect the dominant colour of the fabric. For the other colours such as orange, magenta and blue cyan, the same method is used but the frequency range will be between the primary colours red, blue and green. The result of the recognition for intermediate colours is discussed in Section 3.

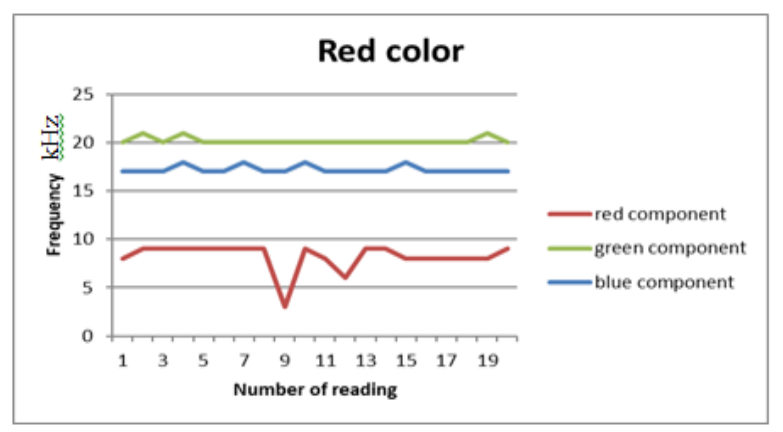

(a)

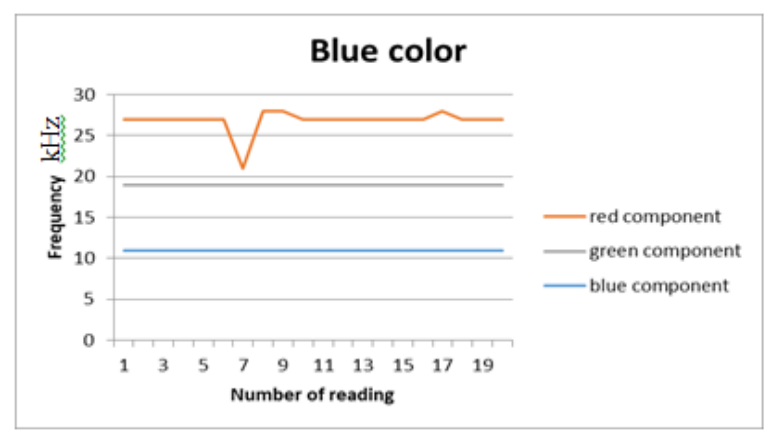

(b)

Figure 7. Initial setup for frequency RGB mapping: (a) Red and (b) Blue

\section{RESULT AND ANALYSIS}

This section will discuss the result of colour recognition experiments for four types of colour. Here the data obtained is frequency according to the frequency-scaling that was implemented in the programming. The plotted graph of the output from the sensor as shown in Figure 8 will be compared to the colour wheel data in Figure 5 in terms of the colour position. The first experiment uses an orange colour object as a testing item. Based on Figure 8(a), the output from the colour sensor shows that when an orange object is put near the device, the frequency output given by the red photodiodes will be lower than that of green and blue photodiodes. As referred to the colour wheel and comparing with the graph, it is expected that the red colour will be dominant followed by green and blue. As shown in Figure 8(b), for cyan colour according to the colour wheel, the expected frequency output will be lower for blue and green as compared to red. This is verified by the graph in Figure 8(b). Then for an object with magenta colour, the frequency output given by the colour sensor will give a lower value for the red, followed by blue and then green as can be seen by Figure 8(c). This is comparable to the colour wheel. Finally, for the blue-magenta, as shown in Figure 8(d), it can be seen that the frequency output of blue photodiodes is lower followed by red and green. This is because the blue colour is a bit more dominant colour for blue-magenta.

As can be seen, when an object placed has a dominant primaries colours under the additive colour system-red, blue and green, those colours will be filtered out and the frequency output will be lower than the other two. As in Figure 8(a), since red is the dominant colour for orange, it will be filtered out and has the lowest frequency compared to blue and green. Here the frequency difference between red and green and blue is about $7 \mathrm{kHz}$ and $9 \mathrm{kHz}$ respectively. For the other additive colours which are yellow, yellow-green, greencyan, blue-cyan and blue-magenta, it can be seen no one type of colour will be dominant or the colour sensor will produce a lower frequency. For secondary colour in the additive colour system, at least two colour can have a low frequency since both dominant colours are detected by the colour sensors. This can be seen in Figure $8(\mathrm{~b})$ and $8(\mathrm{c})$. For cyan, only $5 \mathrm{kHz}$ of difference between blue and green while for magenta only a $2 \mathrm{kHz}$ difference between red and blue. This is logical since cyan is a combination of green and blue and magenta is a combination of red and blue.

As for the tertiary colour of the additives colour system, the frequency output will be lower towards the dominant primary colour (red, blue and green). This can be seen as in Figure $8(\mathrm{~d})$. The frequency recorded in Figure 8(d) (blue-magenta) shows that when compared with the same colour in Figure 8(c) (magenta), the difference of green and blue is about the same $-2 \mathrm{kHz}$ and $4 \mathrm{kHz}$ respectively. But for the red frequency has a big difference of about $10 \mathrm{kHz}$ since less red light is filtered out when tested with a bluemagenta coloured object than red light filtered out for a magenta coloured object. From these experiments, it shows that the programming and mapping of frequency to colour is in a good agreement and can verify the correctness of the colour detection. 


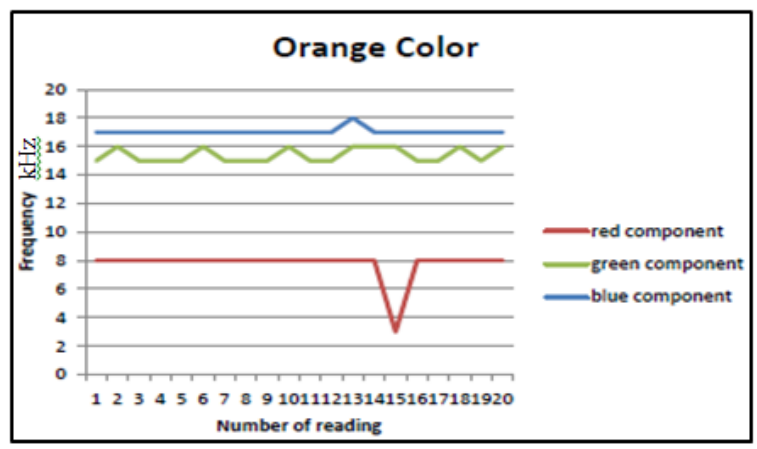

(a)

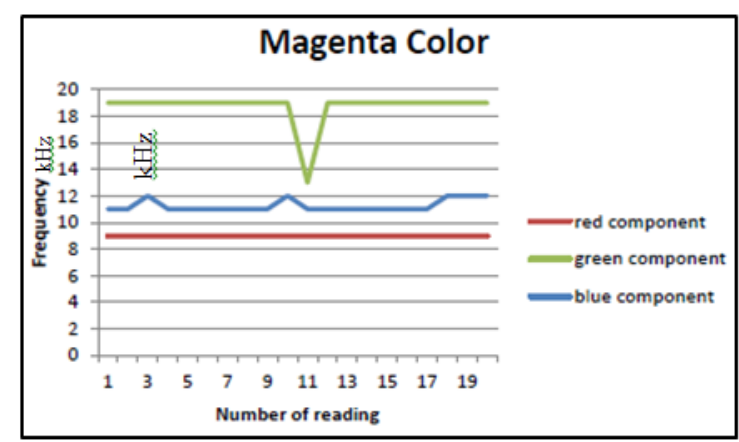

(c)

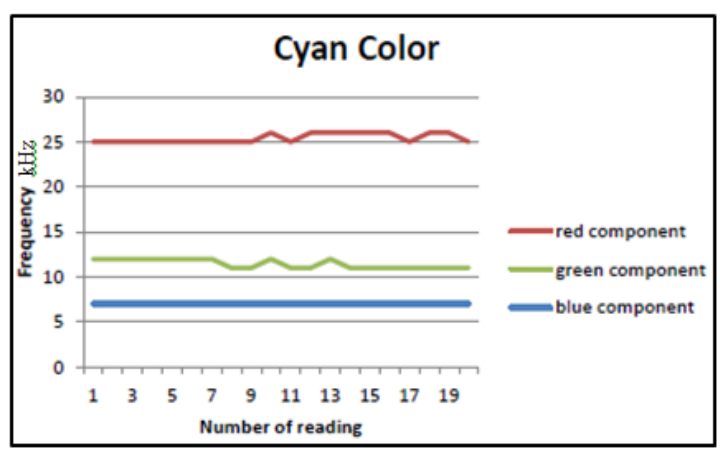

(b)

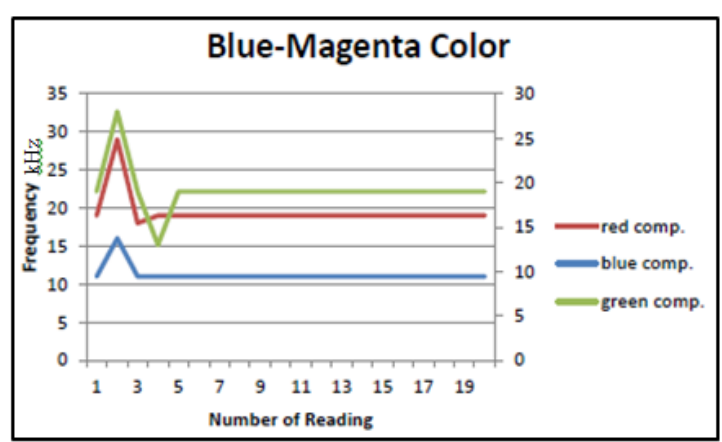

(d)

Figure 8. Frequency against number of reading. (a) Orange. (b) Cyan. (c) Magenta. (d) Blue-magenta colours

\section{CONCLUSION}

According to the result and discussion, the device is beneficial and useful to assist people with visual disability to determine the type of colour in their surrounding object. The design of the device is very user-friendly, practical and cost-effective. The size of the device also is acceptable to hold by human hand. Colour recognizer was successfully developed with medium accuracy and available at affordable price. Also, this device was fabricated using 3D printing and contains an electronic circuit. Then, validation of the colour recogniser has proved the ability of colour recogniser. Lastly, this device will be useful for visually disabled people and can help them to solve their everyday problem.

\section{ACKNOWLEDGEMENTS}

The authors would like to be obliged to Universiti Teknologi Malaysia (UTM) for providing adequate facilities in supporting relevant literatures. In addition, the authors would like to thank Tier 1 UTM vote $16 \mathrm{H} 36$ for the support reviewing this article and financial support.

\section{REFERENCES}

[1] "Visual impairment and blindness". (n.d.). Retrieved May, 15 http://www.who.int/mediacentre/factsheets/fs282/en/

[2] "Blindness and Vision Impairment". Retrieved October, 10 $0^{\text {th }}$, 2018. https://www.who.int/news-room/factsheets/detail/blindness-and-visual-impairment

[3] William B, Gudrun B. (1986). "Colours without sight: A Method for Differentiating Colours in Braille", ETC: A Review of General Semantics, 43(4), 332-335.

[4] Ishaani M, Apoorva M, and Indu S. (2016). "A novel approach to a camera based assistive aid for visually impaired". $3^{\text {rd }}$ International Conference on Recent Advances in Information Technology, Indian School of Mines, Dhanbad, India.

[5] Samara M, AlSadah J, Driche M and Osais Y. (2017). "A colour recognition system for the visually impaired people", $4^{\text {th }}$ IEEE International Conference on Engineering Technologies and Applied Sciences (ICETAS), Salmabad, Bahrain.

[6] Preeyada M, Pratch P, Kittiphong R, and Upady H. (2016). "The talking colour identifying device for the visually impaired", $13^{\text {th }}$ International Conference on Electrical Engineering/Electronics, Computer, Telecommunications and Information Technology, Chiang Mai, Thailand. 
[7] Wahid MA, Ahmad MY, Zain MZM, Saad SM, Hussein M and Ahmad Z. (2018). "A Preliminary study on colour recogniser for visually disabled people". Journal of Telecommunication, Electronic and Computer Engineering, 10(1-15), 139-142.

[8] Thaler S, Milne L, Kish, D, and Goodale M. (n.d). "Shape-specific activation of occipital cortex in an early blind echolocation expert”. Neuropsychologia 51(5), 938-949.

[9] Arnott SN. (2005). "How the blind "see" Braille: lessons from functional magnetic resonance imaging. The Neuroscientist: A Review". Journal Bringing Neurobiology, Neurology and Psychiatry. 11(6), 577-582.

[10] Colour System. (n.d.). Retrieved May 15 ${ }^{\text {th }}, 2018$, from https://www.colormatters.com/color-and-design/color-systems-rgb-and-cmyk

[11] Craig FB. (2006). "Fundamentals of Atmospheric Radiation: An Introduction with 400 Problems". Wiley-VCH. ISBN 3-527-40503-8.

[12] Alfred. (n.d). "Everything you need to know about colour sensors". Retrieved, December $3^{\text {th }}, 2018$ http://www.instructables.com/id/Everything-you-need-to-know-about-colour-sensors/step3/21-Calibration/

[13] M. Samara, J. AlSadah, M. Driche and Y. Osais, "A color recognition system for the visually impaired people," 2017 4th IEEE International Conference on Engineering Technologies and Applied Sciences (ICETAS), Salmabad, 2017, pp. 1-5.

[14] Tcs3200, "Tcs3210 Programmable Color Light-To-Frequency Converter". Retrieved May, 15 2017. https://www.mouser.com/catalog/specsheets/TCS3200-E11.pdf

[15] Dhamini, L. and Bhanuprakash, D., 2018. "Color Recognition System for Visually Impaired Persons". CVR Journal of Science and Technology, 14, pp.11-15.

[16] Kumar, M.A., Jilani, S.A.K., Sreenivasulu, U. and Hussain, S.J., 2015. “Automated Color Recognition System for Visually Challenged and Achromatopsia People using Arduino and Mobile App". International Journal of Advanced Research in Electronics and Communication Engineering, 4(8), pp.2106-2110.

[17] Manaf, Alfa Sheffildi, and Riri Fitri Sari. "Color recognition system with augmented reality concept and finger interaction: Case study for color blind aid system." In 2011 Ninth International Conference on ICT and Knowledge Engineering, pp. 118-123. IEEE, 2012.

[18] Hashim, Nik Nur Wahidah Nik, Tarek Mohamed Bolad, And Noor Hazrin Hany Mohamad Hanif. "Color Recognition Wearable Device Using Machine Learning For Visualy Impaired Person." Iium Engineering Journal 19, No. 2 (2018): 213-220.

[19] Olanrewaju, R.F. and Eniola, F.F., 2016, July. “Automated Bank Note Identification System for Visually Impaired Subjects in Malaysia". In 2016 International Conference on Computer and Communication Engineering (ICCCE) (pp. 115-120). IEEE.

[20] Al-Nabulsi, J., Mesleh, A. and Yunis, A., 2017, October. "Traffic light detection for colorblind individuals". In 2017 IEEE Jordan Conference on Applied Electrical Engineering and Computing Technologies (AEECT), pp. 1-6. IEEE.

[21] Bagwan, S.M.R. and Sankpal, L.J., 2015, September. "VisualPal: A mobile app for object recognition for the visually impaired". In 2015 International Conference on Computer, Communication and Control (IC4), pp. 1-6. IEEE.

[22] Hatasaen, J. and Sunat, K., 2016, July. "A Thai Color Detection Tool for the Blind on Smartphones". In Proceedings of the international Convention on Rehabilitation Engineering \& Assistive Technology (p. 10). Singapore Therapeutic, Assistive \& Rehabilitative Technologies (START) Centre.

[23] Mascetti, S., Ahmetovic, D., Gerino, A., Bernareggi, C., Busso, M. and Rizzi, A., 2016. "Robust traffic lights detection on mobile devices for pedestrians with visual impairment". Computer Vision and Image Understanding, 148, pp.123-135.

[24] Bangar, S., Narkhede, P. and Paranjape, R., 2013. Vocal vision for visually impaired. Int. J. Eng. Sci, 2, pp.1-7.

[25] Albraheem, L., Almotiry, H., Abahussain, H., AlHammad, L., Alshehri, M., AlDosari, R. and Alkathiri, S., 2014, September. "Toward designing efficient application to identify objects for visually impaired". In 2014 IEEE International Conference on Computer and Information Technology, pp. 345-350. IEEE.

\section{BIOGRAPHIES OF AUTHORS}

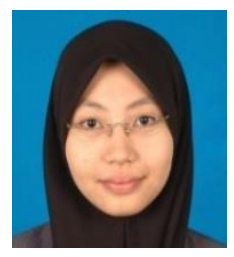

Norazila Othman received her $\mathrm{PhD}$ degree in Applied Aerodynamics from Tokyo Metroplitan University, Tokyo in 2016. Currently, she works as a Senior Lecturer in the Department of Aeronautical, Automotive and Oceanic, School of Mechanical Engineering, Faculty of Engineering, Universiti Teknologi Malaysia (UTM), Malaysia. She teachs applied numerical methods course and applied thermodynamics \& heat transfer course for undergraduate student. Her research interests are in applied aerodynamics, aeronautical design optimization and propulsion analysis related to solid and liquid propellant. 

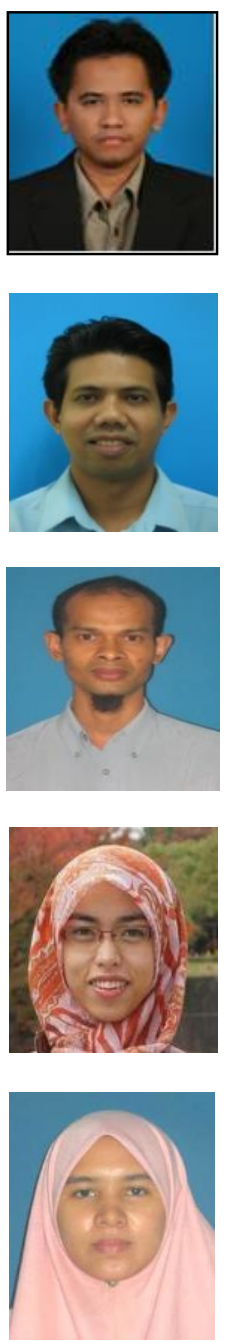

Zarhamdy Md. Zain received his $\mathrm{PhD}$ degree in control system engineering from Universty of Sheffield, UK in 2010. Currently, he works as a Senior Lecturer in the Department of Aeronautical, Automotive and Oceanic, School of Mechanical Engineering, Faculty of Engineering, Universiti Teknologi Malaysia (UTM), Malaysia. He teachs Aircraft Design and static for Aeronautics undergraduate student. His research interests are in mechatronics design, industrial automation and robotics.

Iskandar Shah Ishak received his $\mathrm{PhD}$ degree in Applied Aerodynamics from Universiti Teknologi Malaysia, UTM Johor Bahru in 2012. Currently, he works as a Senior Lecturer in the Department of Applied Mechanics and Design, School of Mechanical Engineering, Faculty of Engineering, Universiti Teknologi Malaysia (UTM), Malaysia. He teachs Mechatronics and control courses for Mechanical undergraduate student. His research interests are in helicopter aerodynamics and wind tunnel experiments.

Abdul Rahim Abu Bakar received his $\mathrm{PhD}$ degree in automotive system from Universiti of Liverpool, UK in 2004. Currently, he works as a Senior Lecturer in the Department of Aeronautical, Automotive and Oceanic, School of Mechanical Engineering, Faculty of Engineering, Universiti Teknologi Malaysia (UTM), Malaysia. His research interest is automotive vibration and its applications.

Mastura $\mathrm{Ab}$ Wahid received her $\mathrm{PhD}$ degree in Aircraft control, navigation and guidance from Ecole Nationale d'Aéronautique Civile, Toulouse, France in 2015. Currently, she works as a Senior Lecturer in the Department of Aeronautical, Automotive and Oceanic, School of Mechanical Engineering, Faculty of Engineering, UniversitiTeknologi Malaysia (UTM), Malaysia. Her research interests are in guidance and control and also air traffic management.

Maziah Mohamad received her PhD degree in automatic control and system engineering from Universiti of Sheffield, UK in 2011. Currently, she works as a Senior Lecturer in the Department of Applied Mechanics and Design, School of Mechanical Engineering, Faculty of Engineering, Universiti Teknologi Malaysia (UTM), Malaysia. Her research interests are in field of control engineering and artificial intelligent algorithm. 